\title{
Relationship between Repeated Sprint Ability and Aerobic Capacity in Professional Soccer Players
}

\author{
Rhys M. Jones, ${ }^{1}$ Christian C. Cook, ${ }^{2}$ Liam P. Kilduff, ${ }^{1}$ \\ Zoran Milanović, ${ }^{3}$ Nic James, ${ }^{4}$ Goran Sporiš, ${ }^{5}$ Bruno Fiorentini, ${ }^{6}$ \\ Fredi Fiorentini, ${ }^{7}$ Anthony Turner, ${ }^{4}$ and Goran Vučkovićc ${ }^{8}$ \\ ${ }^{1}$ Sport and Exercise Science Research Centre, Talbot Building, Swansea University, Swansea SA2 8PP, UK \\ ${ }^{2}$ UK Sport Council, 40 Bernard Street, London, UK \\ ${ }^{3}$ Faculty of Sport and Physical Education, University of Nis, Čarnojevićeva 10a, 18000 Nis, Serbia \\ ${ }^{4}$ London Sport Institute, Middlesex University, London NW4 4BT, UK \\ ${ }^{5}$ Faculty of Kinesiology, University of Zagreb, 10000 Zagreb, Croatia \\ ${ }^{6}$ Ken Blanchard College of Business, Grand Canyon University, AZ 85017, USA \\ ${ }^{7}$ Faculty of Kinesiology, University of Split, 21000 Split, Croatia \\ ${ }^{8}$ Faculty of Sport, University of Ljubljana, 1000 Ljubljana, Slovenia
}

Correspondence should be addressed to Goran Vučković; goran.vuckovic@fsp.uni-lj.si

Received 5 July 2013; Accepted 27 July 2013

Academic Editors: C. C. Chase Jr and D. S. Gardner

Copyright (c) 2013 Rhys M. Jones et al. This is an open access article distributed under the Creative Commons Attribution License, which permits unrestricted use, distribution, and reproduction in any medium, provided the original work is properly cited.

\begin{abstract}
Aim. The aim of the present study was to investigate the relationship between maximal aerobic capacity $\left(\mathrm{VO}_{2 \max }\right)$ and repeated sprint ability (RSA) in a group of professional soccer players. Methods. Forty-one professional soccer players (age $23 \pm 4$ yrs, height $180.0 \pm 5.3 \mathrm{~cm}$, weight $79.6 \pm 5.3 \mathrm{~kg}$ ) were required to perform tests to assess $\mathrm{RSA}$ and $\mathrm{VO}_{2 \max }$ on two separate days with at least $48 \mathrm{hr}$ rest between testing sessions. Each player performed a treadmill test to determine their $\mathrm{VO}_{2 \max }$ and a test for RSA involving the players completing $6 \times 40 \mathrm{~m}$ sprints (turn after $20 \mathrm{~m}$ ) with $20 \mathrm{~s}$ active recovery between each sprint. Results. There was a significant negative correlation between body mass normalised $\mathrm{VO}_{2 \max }$ and mean sprint time $\left(\mathrm{RSA}_{\text {mean }}\right)(r=-0.655 ; P<0.01)$ and total sprint time $\left(\mathrm{RSA}_{\text {total }}\right)(r=-0.591, P<0.01)$. Conclusion. Results of the current study indicate that $\mathrm{VO}_{2 \max }$ is one important factor aiding soccer players in the recovery from repeated sprint type activities.
\end{abstract}

\section{Introduction}

The ability to perform (and recover from) repeated high intensity activities over a prolonged period of time coupled with a good aerobic capacity is deemed essential physiological requirements for success in soccer [1]. For example, maximal aerobic capacity $\left(\mathrm{VO}_{2 \max }\right)$ in soccer squads has been correlated to playing standard [2] and league position [3], suggesting that higher $\mathrm{VO}_{2 \max }$ values enable players to have a physiological advantage to perform in elite soccer. Further support of this fact can be taken from the work of Rampinini et al. [4] who reported significant correlations between peak speed reached during an incremental field test and total distance covered $(r=0.58)$, high intensity running $(r=0.65)$, and very high intensity running during competitive games $(r=0.64)$.

In addition to the importance of having a good aerobic capacity, players also perform approximately 150-250 brief intense bouts of activity during a game with these bouts of activity contributing to the more crucial moments of the game such as winning possession, shooting, and chasing a player to tackle [5]. Using match analysis technology, it has been demonstrated that international players perform $28 \%$ more high intensity running and $58 \%$ more sprinting than professional players of a lower standard [5]. The amount of high intensity exercise performed (and recovery from it) is one factor that helps distinguish top-class players from players of a lower standard [1]. Players who can regularly 
perform repeat sprint efforts at the same or very similar intensity and quality are therefore likely to perform better over extended periods of time [4]. The extent to which an individual can maintain their sprint performance is known as "repeated sprint ability" (RSA) [6] which is largely dependent on the extent of Phosphocreatine (PCr) resynthesis [7] and the removal of hydrogen ions $\left(\mathrm{H}^{+}\right)$from the muscle during recovery between bouts. It has previously been proposed that an individual's RSA may be aided by their aerobic capacity [8] as an enhanced aerobic capacity may increase the ability to tolerate, remove, and buffer $\mathrm{H}^{+}$from the working muscle [9] and also enhance PCr and adenosine triphosphate (ATP) resynthesis from inorganic phosphates postexercise [10].

To date, only a limited number of studies have examined the relationship between $\mathrm{VO}_{2 \max }$ and RSA with one study reporting a significant relationship between $\mathrm{VO}_{2 \max }$ and total sprint time $\left(\mathrm{RSA}_{\text {total }} ; r=-0.49\right)$ in a group of amateur team sport and racquet athletes [6]. Additional research examining the existence of a relationship between RSA and $\mathrm{VO}_{2 \max }$ in elite athletes has produced equivocal findings [8, 11-14]. To the authors knowledge, only one paper has found a relationship between RSA and $\mathrm{VO}_{2 \max }$ using elite soccer players [8]. However, a major limitation of this work and other research which has found no significant relationship between RSA and $\mathrm{VO}_{2 \text { max }}$ is the lack of reliability and logical, construct, and criterion validity of RSA protocols in reference to the sport in question. Furthermore, studies have indirectly determined $\mathrm{VO}_{2 \max }$ using field tests [11,14] which is likely to result in up to $10 \pm 15 \%$ inaccuracy level [15]. Recently, a protocol to assess RSA specific to the demands of elite soccer has been implemented which has demonstrated reliability and validity $[4,16]$. The repeated sprint protocol involves six $40 \mathrm{~m}$ shuttle sprints separated by $20 \mathrm{~s}$ passive recovery, with each sprint including a $180^{\circ}$ turn after $20 \mathrm{~m}$, thus including a deceleration, turn, and acceleration, specific to the demands of soccer. Furthermore, mean repeated sprint time $\left(\mathrm{RSA}_{\text {mean }}\right)$ has been reported as $7.25 \pm 0.17 \mathrm{~s}$ (range 6.95-7.50 s) in elite soccer players; thus work to rest ratios of $1: 2.8$ are achieved with the mean total duration lasting $143.5 \mathrm{~s}$, which is similar to the most demanding phases of a soccer match giving the protocol logical validity [4].

In light of the above the present study aimed to determine whether a relationship exists between RSA and $\mathrm{VO}_{2 \max }$ in professional soccer players. We hypothesized that $\mathrm{VO}_{2 \max }$ would be associated with RSA in professional soccer players.

\section{Methods}

2.1. Subjects. Forty-one professional soccer players (age $23 \pm$ 4 yrs, height $180.0 \pm 5.3 \mathrm{~cm}$, weight $79.6 \pm 5.3 \mathrm{~kg}$ ), from whom written informed consent had been obtained, volunteered to take part in the present study which was approved by the universities ethics committee. Players refrained from heavy training for the 2 days prior to testing. During the 2 hours before testing they were only allowed ad libitum water intake with subjects consuming a light meal 3 hours before testing. Players were accustomed to the testing procedures
TABLE 1: Incremental treadmill test protocol.

\begin{tabular}{lcc}
\hline Time $(\mathrm{min})$ & Speed $\left(\mathrm{km} \cdot \mathrm{h}^{-1}\right)$ & Gradient $(\%)$ \\
\hline $0-2$ & 10 & 0 \\
$2-4$ & 12 & 0 \\
$4-6$ & 14 & +2.5 \\
$6-8$ & 16 & +5.0 \\
$8-10$ & 18 & +5.0 \\
$10-12$ & 20 & +5.0 \\
\hline
\end{tabular}

and had performed $\mathrm{VO}_{2 \max }$ and tests for RSA on a number of occasions prior to the commencement of the present study. The present study was performed at the end of preseason training for the season beginning 2008-2009.

2.2. Experimental Procedures. For both tests, players were asked to refrain from alcohol and caffeine $24 \mathrm{hrs}$ prior to testing reducing the possibility of dehydration affecting performance. During all testing players were given encouragement from testing and club staff.

\subsection{Measurements}

2.3.1. Maximal Aerobic Uptake $\left(\mathrm{VO}_{2 \max }\right)$. $\mathrm{VO}_{2 \max }$ was determined following an incremental treadmill run to fatigue (Woodway Ergo ELG 55 treadmill; Woodway GmbH, Weil am Rhein, Germany) with gas exchange and ventilatory variables being analysed breath-by-breath using a calibrated computer-based exercise system (Jaeger Oxycon Pro online gas analyser; Erich Jaeger $\mathrm{GmbH}$, Hoechberg, Germany). The $\mathrm{CO}_{2}$ and $\mathrm{O}_{2}$ analyzers were calibrated before each test using a two-point measure: a calibration gas $\left(\mathrm{CO}_{2} 5 \%\right.$, $\mathrm{O}_{2} 16 \%, \mathrm{~N}_{2}$ balance) and a reference gas (room air after ATPS (ambient temperature and pressure, saturated) to STPD (standard temperature and pressure, dry)). Heart rate (HR) was continuously recorded (Polar; Lake Success, NY, USA).

Following the measurement of each subject's stature (Holtain Stadiometer; Holtain Ltd., Crymych, Wales) and body mass (Seca 888 Class III floor scale; Seca, Birmingham, UK) subjects performed 5 min light running at a self-selected pace. The protocol for the exercise test was preinstalled to allow automatic and accurate increments in speed $\left(\mathrm{km} \cdot \mathrm{h}^{-1}\right)$ and gradient (\%) (Table 1).

2.3.2. Repeated Sprint Ability (RSA). The RSA protocol required each subject to complete six $40 \mathrm{~m}$ sprints with $20 \mathrm{~s}$ active recovery between each sprint. A sprint consisted of the subject taking up a start position with their leading foot placed $30 \mathrm{~cm}$ behind the start line, which was instrumented with timing gates (Brower timing systems; Draper, UT, USA). When ready the subject sprinted through the timing gates (starting the timing) to a line marked on the track $20 \mathrm{~m}$ from the start line. Once the subject's foot touched on the line at the $20 \mathrm{~m}$ mark, they turned (as fast as possible) and sprinted back. Timing stopped when the subject recrossed the start line.

Prior to testing, subjects performed a $10 \mathrm{~min}$ warmup which included light running and dynamic stretching, 


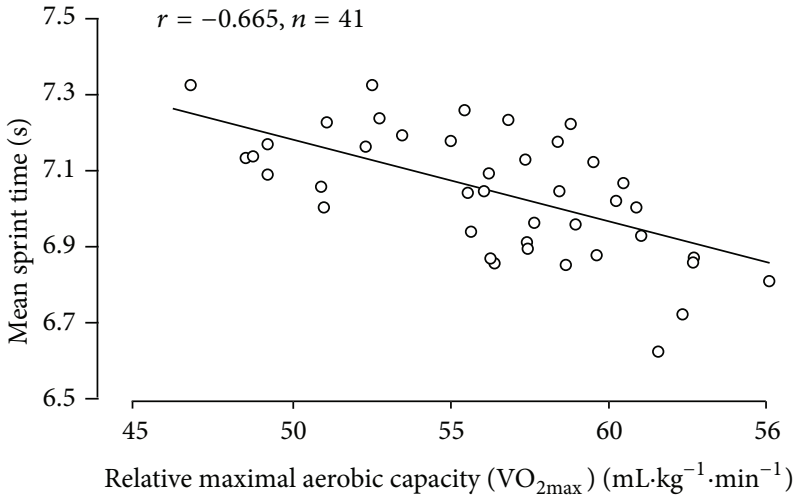

(a)

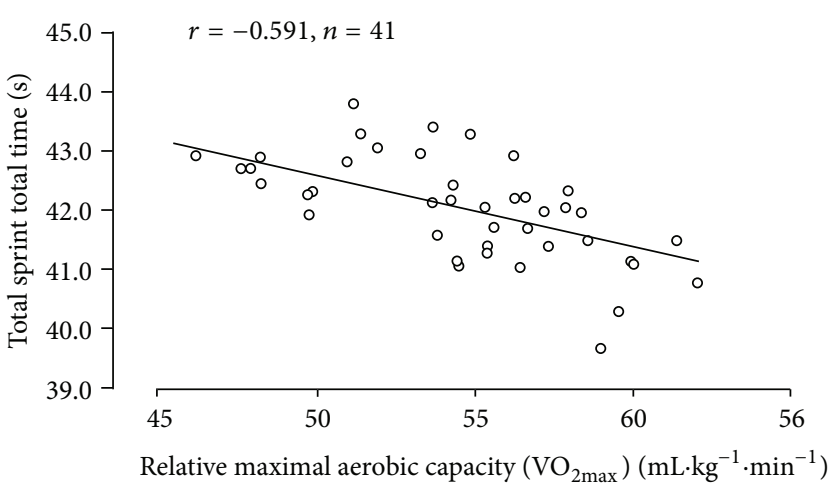

(b)

FIGURE 1: Relationship between relative aerobic capacity and mean sprint time (a) and total sprint time (b).

followed by several maximal sprints over $20 \mathrm{~m}$. Three minutes following the warm-up, players underwent 2 preliminary $40 \mathrm{~m}$ shuttle sprints, with the fastest time used as a criterion score for the repeated sprint test. Five minutes from the second criterion run, subjects began the RSA test. If the first sprint during the RSA test $2.5 \%$ or more slow than the criterion time, then the repeated sprint performance was deemed not to be maximal and discarded from analysis. Performance variables analysed from the RSA test were total sprint time $\left(\mathrm{RSA}_{\text {total }}\right)$ (time for all 6 sprints combined) and mean sprint time ( $\mathrm{RSA}_{\text {mean }}$ ) (average over the 6 sprints).

The reliability (typical error expressed as a coefficient of variation) for $\mathrm{RSA}_{\text {mean }}$ has been reported to be $0.8 \%$ [17]. In addition the validity of the test was assessed and significant correlations between $\mathrm{RSA}_{\text {mean }}$ time and match performance variables (i.e., very high intensity running and sprinting distance) as assessed match analysis systems [4].

2.4. Statistical Analysis. Data are presented as the mean \pm standard deviation (SD). A two-tailed Pearson's productmoment correlation was used to determine the strength and directionality of the relationship between $\mathrm{VO}_{2 \max }$ and RSA $\left(\mathrm{RSA}_{\text {mean }}, \mathrm{RSA}_{\text {total }}\right)$. The coefficient of determination $\left(r^{2}\right)$ was used to examine the amount of explained variance between tests. The level of significance was set at $P<0.05$.

\section{Results}

The correlation coefficients between absolute and relative $\mathrm{VO}_{2 \max }$ and the RSA performance indicators are summarised in Table 2 . There were significant negative correlations between relative $\mathrm{VO}_{2 \max }$ and $\mathrm{RSA}_{\text {mean }}(r=-0.655$, $P<0.01$; Figure 1(a)) and $\mathrm{RSA}_{\text {total }}(r=-0.591, P<0.01$; Figure 1(b)). However, no such correlation existed between absolute $\mathrm{VO}_{2 \text { max }}$ and $\mathrm{RSA}_{\text {mean }}$ or $\mathrm{RSA}_{\text {total }}$ (Table 2).
TABlE 2: Correlation coefficients between maximal oxygen uptake and repeated sprint ability test performance indices.

\begin{tabular}{llc}
\hline \multirow{2}{*}{ Repeated sprint ability } & \multicolumn{2}{c}{ Maximal oxygen uptake } \\
& Relative & Absolute \\
\hline Mean sprint time (s) & $-0.655^{*}$ & -0.068 \\
Total sprint time (s) & $-0.591^{*}$ & 0.033 \\
\hline
\end{tabular}

*indicates $P<0.05$.

\section{Discussion}

The present study examined the relationships between aerobic capacity and repeated sprint ability in a group of professional soccer players. The key finding from the present study was that significant moderate negative correlations were found between $\mathrm{VO}_{2 \max }\left(\mathrm{mL} \cdot \mathrm{kg} \cdot \mathrm{min}^{-1}\right)$ and RSA expressed as $\mathrm{RSA}_{\text {mean }}(r=-0.655, P<0.01)$ and $\mathrm{RSA}_{\text {total }}(r=$ $-0.591, P<0.01)$. The present study supports the theory that aerobic capacity is an important factor influencing recovery from RSA in elite soccer. The ability of an enhanced $\mathrm{VO}_{2} \max$ to improve recovery between repeated bouts of maximal sprinting is likely related to the ability to tolerate, remove, and buffer hydrogen ions $\left(\mathrm{H}^{+}\right)$from the working muscle [9] while efficiently restoring PCr and ATP stores from inorganic phosphates postexercise [7]. Previous research has shown that the extent of $\mathrm{PCr}$ degradation and $\mathrm{H}^{+}$accumulation, which increases with repeated bouts of maximal exercise [18], is associated with muscular fatigue in soccer players. Various mechanisms could be proposed; for example, an individual with a higher $\mathrm{VO}_{2 \max }$ may exhibit an increased mitochondrial number, size, and surface area [19] which may allow greater movement of pyruvate into the mitochondria. Increased concentrations of aerobic enzymes will enhance the capacity to generate ATP by oxidative phosphorylation. Furthermore, individuals with an increased $\mathrm{VO}_{2 \max }$ may have increased myoglobin concentrations [20]. This would enhance the ability of the skeletal muscle to move oxygen 
from the muscle cell membrane to the mitochondria and will increase the magnitude of myoglobin oxygen stores, thus increasing the delivery of oxygen $\left(\mathrm{O}_{2}\right)$ to the mitochondria at the onset of exercise. Furthermore, an elevated $\mathrm{VO}_{2 \max }$ has been proposed to directly enhance lactate removal and PCr restoration, thus enhancing power and force recovery. Lactate removal is dependent on a series of events with approximately $65 \%$ of the lactate converted to pyruvate by lactate dehydrogenase (LDH), which then undergoes aerobic degradation via the krebs cycle and electron transport system with the nonconverted 35\% secreted as urine and sweat or converted to protein [21]. If an enhanced $\mathrm{VO}_{2 \max }$ is associated with enhanced aerobic enzyme concentrations and an increased mitochondrial surface area providing greater transport sites for pyruvate [19], it is likely to enhance lactate removal.

Previous research supporting the relationship between $\mathrm{VO}_{2 \text { max }}$ and RSA has been limited in elite athletes. For a study to assess RSA for a specific sport, the protocol used should be specific to the physiological response and activity pattern of the most intense repeated sprint activity observed from the sport (logical validity), should be reliable, and should have construct and criterion validity [11]. Studies that have utilised indirect methods of determining $\mathrm{VO}_{2 \max }[11,14]$ including the multistage fitness test which is likely to have 10$15 \%$ inaccuracy are therefore inaccurate when assessing the relationship between RSA and $\mathrm{VO}_{2 \max }$.

In contrast with the results of this study, some studies $[8,22]$ have reported that $\mathrm{VO}_{2 \max }$ is a poor indicator of RSA $(r=0.09-0.03)$. This may be explained by differences in the type of protocol used for the RSA test [23] where $\mathrm{VO}_{2 \text { max }}$ has not been reported to be related to RSA when sprints of less than 40 meters or 6 seconds have been used. Furthermore, RSA may also be influenced by the training status of the subjects [24]. Da Silva et al. [23] have concluded that the minimum velocity needed to reach $\mathrm{VO}_{2 \max }$ and velocity at the onset of blood-lactate accumulation were more strongly correlated with RSA indices than $\mathrm{VO}_{2 \text { max }}$.

A key physiological requirement of most intermittent team sports is the ability to repeatedly produce short, maximal effort activities with incomplete (and varying) recovery (repeated sprint ability (RSA)). Soccer players are required to frequently produce brief intense actions (150200 per game) [5] which constitute some of the most crucial moments of the game [1], and time motion analysis has demonstrated that players in all playing positions experience a significant decline in the number of high intensity actions performed across a match [5]. Furthermore, the ability to limit this decline in high intensity activities appears to be associated with playing standard with international players performing 28\% more high intensity running and 58\% more sprinting than professional players of a lower standard [5]. This suggests the ability to recover between bouts of high intensity activity may be a key determinant of performance in elite soccer. However, the physiological mechanism facilitating recovery between repeated sprints has previously been unclear. With aerobic contribution increasing with the number of repeated bouts and oxygen uptake potential contributing to recovery between maximal bouts of sprinting, it has previously been proposed that an enhanced aerobic capacity may influence recovery between bouts of high intensity activity [8]. Further support for this theory has come from match analysis data in elite soccer which has shown that improvements in $\mathrm{VO}_{2 \max }\left(58.1 \pm 4.5 \mathrm{~mL} \cdot \mathrm{kg} \cdot \mathrm{min}^{-1}\right.$ to $64.3 \pm 3.9 \mathrm{~mL} \cdot \mathrm{kg} \cdot \mathrm{min}^{-1}$ ) following 8 weeks of interval training lead to significant improvements in distance covered during games (20\%), number of sprints (100\%), number of involvements with the ball (24\%), and work intensity measured as percentage of maximal heart rate $(82.7 \pm 3.4 \%$ to $85.6 \pm 3.1 \%$ [25]. Furthermore, players with greater $\mathrm{VO}_{2 \max }$ values have been found to cover greater total distances and distances at high intensity during a match [25]. Thus match analysis data suggests that an improved $\mathrm{VO}_{2 \max }$ gives an enhanced potential to recover between bouts of maximal intensity activity allowing greater distances at a higher intensity to be covered during a match.

To the authors knowledge, the present study is the first to assess the relationship between RSA and $\mathrm{VO}_{2 \max }$ using an RSA protocol shown to be reliable and valid, while determining $\mathrm{VO}_{2}$ max directly from breath-by-breath analysis taken during an incremental treadmill run to fatigue. As a consequence the finding from the present study is to date the most conclusive evidence in the assessment of the relationship between RSA and $\mathrm{VO}_{2 \text { max }}$ in elite athletes.

A limitation of this study is that the minimum velocity needed to reach $\mathrm{VO}_{2 \max }$ and velocity at the onset of bloodlactate accumulation were not assessed. Also, we did not assess anaerobic capacity and its relationship with repeated sprint ability in professional soccer players. Future study is required to document relationship between aerobic (velocity needed to reach $\mathrm{VO}_{2 \text { max }}$ and velocity at the onset of bloodlactate accumulation) and anaerobic capacity at the one side and RSA at the other side and determine which of them are better predictor of RSA in professional soccer players.

In conclusion, the results from the present study suggest that, in professional soccer players, there is a relationship between RSA and relative $\mathrm{VO}_{2 \text { max }}$. This suggests that, to improve RSA, it is important to implement specific soccer training targeting aerobic components.

\section{Disclosure}

The authors declare no financial support or relationship that may pose conflict of interests.

\section{References}

[1] M. Svensson and B. Drust, "Testing soccer players," Journal of Sports Sciences, vol. 23, no. 6, pp. 601-618, 2005.

[2] Á. Árnason, S. B. Sigurdsson, A. Gudmundsson, I. Holme, L. Engebretsen, and R. Bahr, "Physical fitness, injuries, and team performance in soccer," Medicine and Science in Sports and Exercise, vol. 36, no. 2, pp. 278-285, 2004.

[3] P. Apor, "Successful formulae for fitness training," in Science and Football London: E \& FN Spon, 1988.

[4] E. Rampinini, D. Bishop, S. M. Marcora, D. Ferrari Bravo, R. Sassi, and F. M. Impellizzeri, "Validity of simple field tests 
as indicators of match-related physical performance in toplevel professional soccer players," International Journal of Sports Medicine, vol. 28, no. 3, pp. 228-235, 2007.

[5] M. Mohr, P. Krustrup, and J. Bangsbo, "Match performance of high-standard soccer players with special reference to development of fatigue," Journal of Sports Sciences, vol. 21, no. 7, pp. 519528, 2003.

[6] B. Dawson, M. Fitzsimons, and D. Ward, "The relationship of repeated sprint ability to aerobic power and performance measures of anaerobic work capacity and power," Australian Journal of Science and Medicine in Sport, vol. 25, no. 4, pp. 88-93, 1993.

[7] G. C. Bogdanis, M. E. Nevill, L. H. Boobis, and H. K. A. Lakomy, "Contribution of phosphocreatine and aerobic metabolism to energy supply during repeated sprint exercise," Journal of Applied Physiology, vol. 80, no. 3, pp. 876-884, 1996.

[8] A. R. Aziz, M. Chia, and K. C. Teh, “The relationship between maximal oxygen uptake and repeated sprint performance indices in field hockey and soccer players," Journal of Sports Medicine and Physical Fitness, vol. 40, no. 3, pp. 195-200, 2000.

[9] K. Sahlin and J. Henriksson, "Buffer capacity and lactate accumulation in skeletal muscle of trained and untrained men," Acta Physiologica Scandinavica, vol. 122, no. 3, pp. 331-339, 1984.

[10] R. C. Harris, R. H. T. Edwards, and E. Hultman, "The time course of phosphorylcreatine resynthesis during recovery of the quadriceps muscle in man," Pflügers Archiv European Journal of Physiology, vol. 367, no. 2, pp. 137-142, 1976.

[11] A. R. Aziz, S. Mukherjee, M. Y. H. Chia, and K. C. Teh, "Validity of the running repeated sprint ability test among playing positions and level of competitiveness in trained soccer players," International Journal of Sports Medicine, vol. 29, no. 10, pp. 833-838, 2008.

[12] G. J. Bell, G. D. Snydmiller, D. S. Davies, and H. A. Quinney, "Relationship between aerobic fitness and metabolic recovery from intermittent exercise in endurance athletes," Canadian Journal of Applied Physiology, vol. 22, no. 1, pp. 78-85, 1997.

[13] J. R. Hoffman, S. Epstein, M. Einbinder, and Y. Weinstein, "The influence of aerobic capacity on anaerobic performance and recovery indices in basketball players," The Journal of Strength \& Conditioning Research, vol. 13, no. 4, p. 407, 1999.

[14] C. B. Wragg, N. S. Maxwell, and J. H. Doust, "Evaluation of the reliability and validity of a soccer-specific field test of repeated sprint ability," European Journal of Applied Physiology, vol. 83, no. 1, pp. 77-83, 2000.

[15] P. O. Astrand, Textbook of Work Physiology-4th: Physiological Bases of Exercise, Human Kinetics, 2003.

[16] F. M. Impellizzeri, E. Rampinini, C. Castagna et al., "Validity of a repeated-sprint test for football," International Journal of Sports Medicine, vol. 29, no. 11, pp. 899-905, 2008.

[17] D. Ferrari Bravo, E. Rampinini, R. Sassi et al., "Ecological validity of a repeated sprint ability test and its reproducibility in soccer," in Proceedings of the 10th Congress of the European College of Sport Science, Belgrade, Serbia, 2005, Book of Abstract.

[18] P. D. Balsom, J. Y. Seger, B. Sjödin, and B. Ekblom, "Maximalintensity intermittent exercise: effect of recovery duration," International Journal of Sports Medicine, vol. 13, no. 7, pp. 528533, 1992.

[19] J. O. Holloszy and E. F. Coyle, "Adaptations of skeletal muscle to endurance exercise and their metabolic consequences," Journal of Applied Physiology Respiratory Environmental and Exercise Physiology, vol. 56, no. 4, pp. 831-838, 1984.
[20] B. Saltin and L. B. Rowell, "Functional adaptations to physical activity and inactivity," Federation Proceedings, vol. 39, no. 5, pp. 1506-1513, 1980.

[21] W. S. Parkhouse and D. C. McKenzie, "Possible contribution of skeletal muscle buffers to enhanced anaerobic performance: a brief review," Medicine and Science in Sports and Exercise, vol. 16, no. 4, pp. 328-338, 1984.

[22] G. Wadley and P. le Rossignol, "The relationship between repeated sprint ability and the aerobic and anaerobic energy systems," Journal of Science and Medicine in Sport, vol. 1, no. 2, pp. 100-110, 1998.

[23] J. F. da Silva, L. G. A. Guglielmo, and D. Bishop, "Relationship between different measures of aerobic fitness and repeatedsprint ability in elite soccer players," Journal of Strength and Conditioning Research, vol. 24, no. 8, pp. 2115-2121, 2010.

[24] M. Spencer, D. Bishop, B. Dawson, and C. Goodman, "Physiological and metabolic responses of repeated-sprint activities: specific to field-based team sports," Sports Medicine, vol. 35, no. 12, pp. 1025-1044, 2005.

[25] J. Helgerud, L. C. Engen, U. Wisløff, and J. Hoff, "Aerobic endurance training improves soccer performance," Medicine and Science in Sports and Exercise, vol. 33, no. 11, pp. 1925-1931, 2001. 

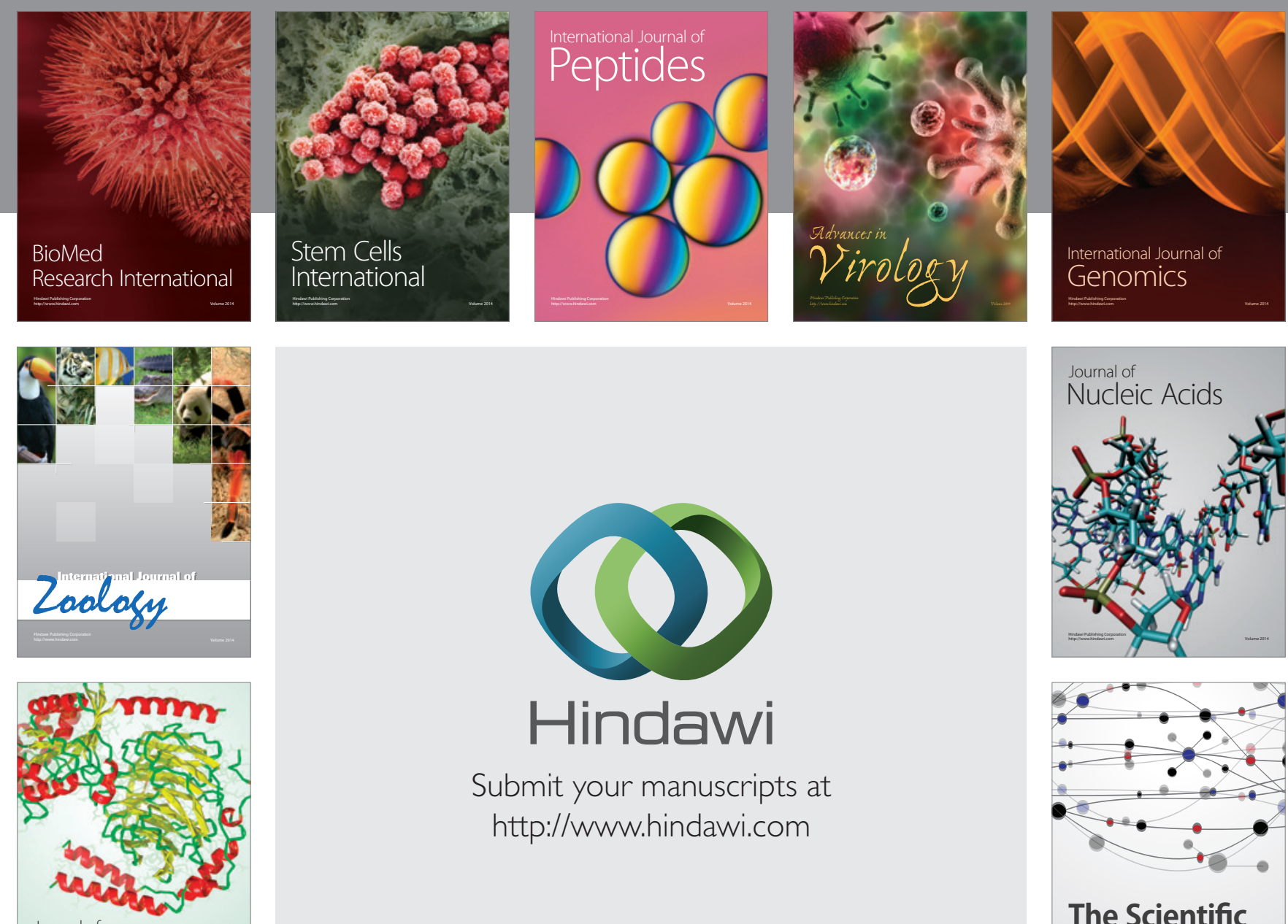

Submit your manuscripts at

http://www.hindawi.com

Journal of
Signal Transduction
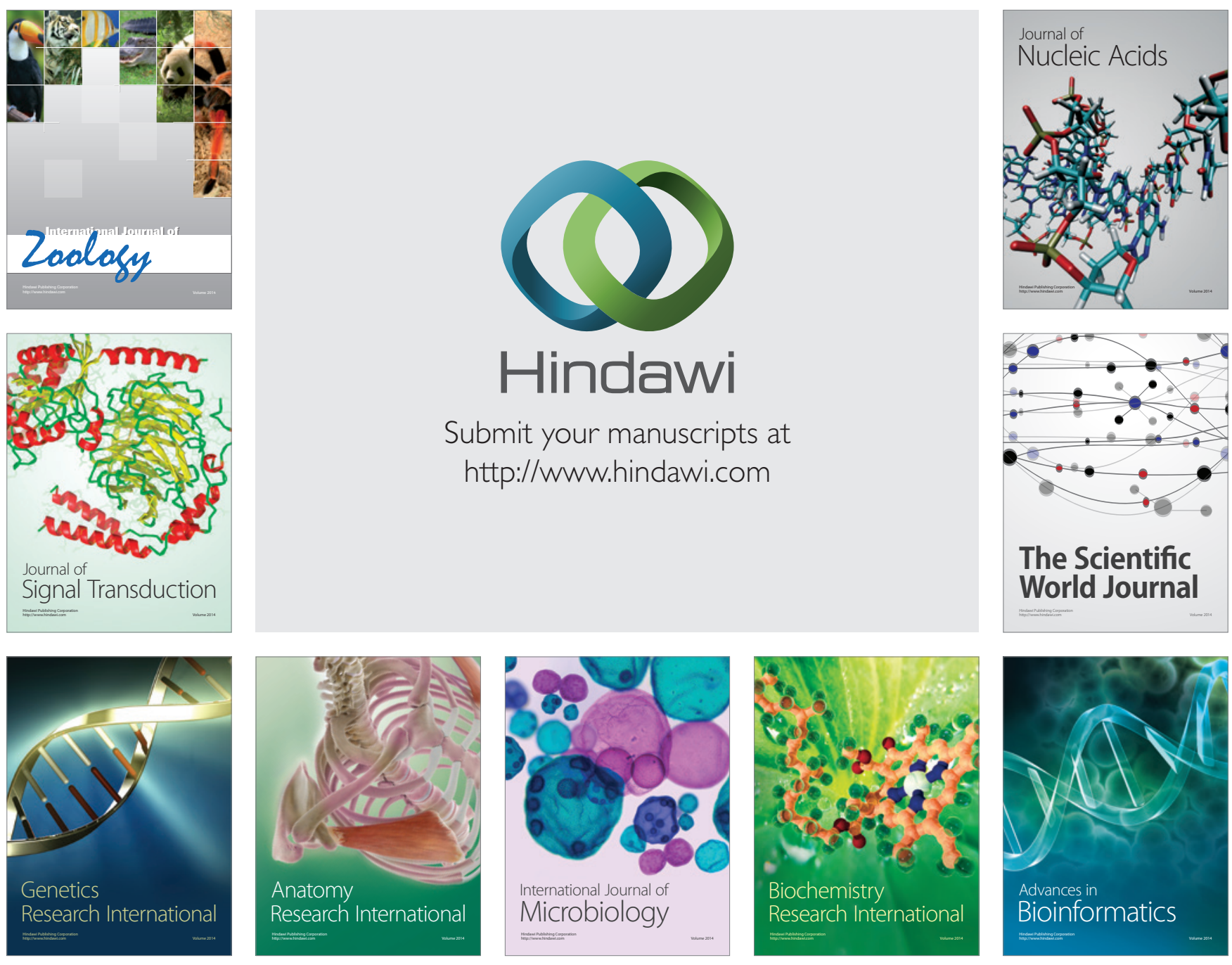

The Scientific World Journal
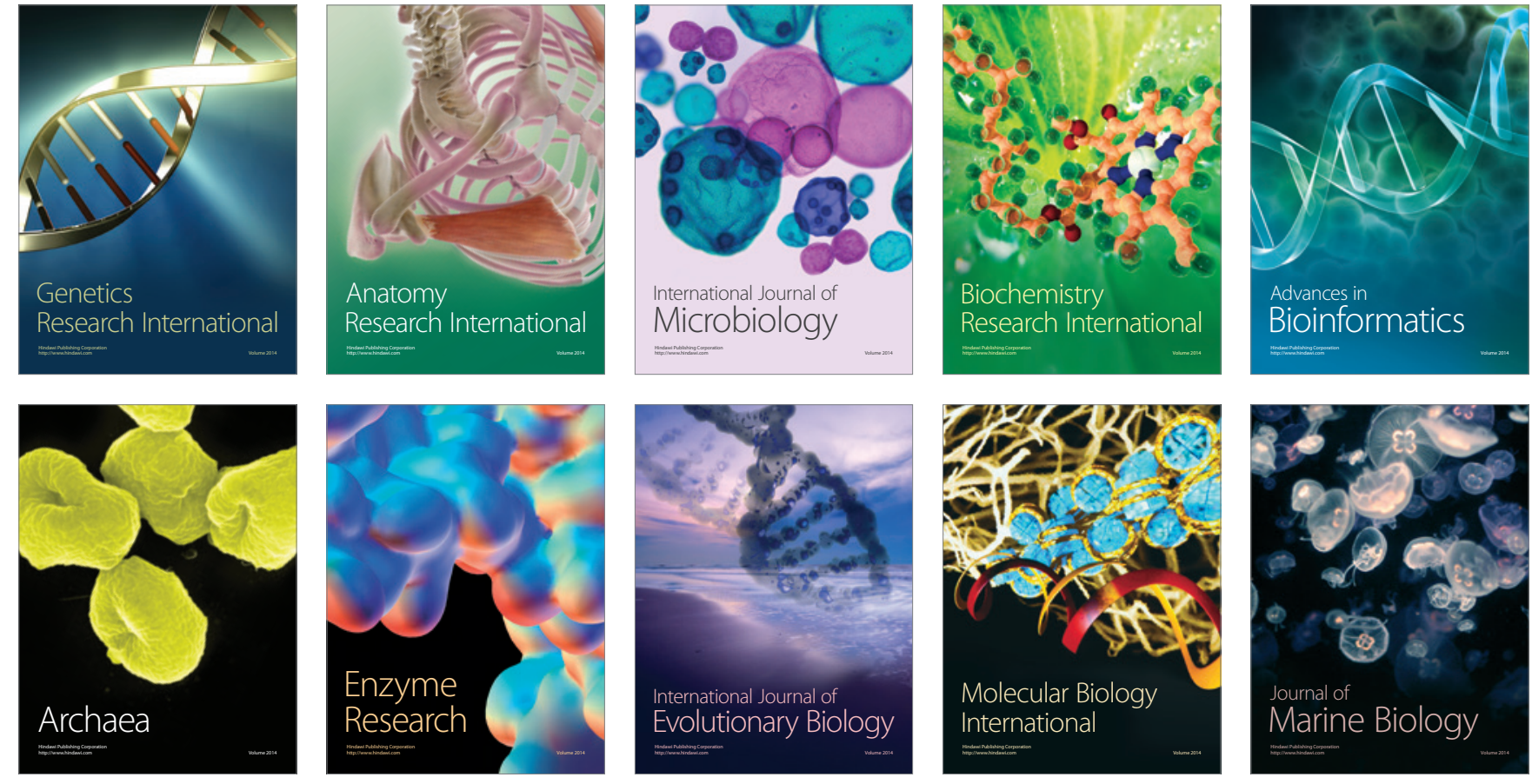